\title{
SIMULATION OF VELOCITY PROFILE INSIDE TURBULENT BOUNDARY LAYER
}

\author{
Karlo T. Raić * \\ University of Belgrade, Faculty of Technology and Metallurgy, Karnegijeva 411120 \\ Belgrade, Serbia
}

Received 10.04.2020

Accepted 10.04.2020

\begin{abstract}
The second-order differential equation with a general polynomial solution [1], is adapted for simulation of complex velocity profile inside the turbulent boundary layer. Consequently, the simulation strategy is suggested.
\end{abstract}

Keywords: velocity profile; turbulent boundary layer; second-order differential equation; polynomial solution.

\section{Introduction}

Turbulent flows are demanding for simulation due to: (i) unsteady aperiodic motion, (ii) fluid properties that exhibit 3D random spatial variations, (iii) strong dependence from initial conditions, and (iv) a wide range of scales (eddies). In other words, the turbulent simulation always has to be three-dimensional, time accurate with extremely fine grids [2-4].

Direct Numerical Simulation (DNS) under the time-dependent Navier-Stokes equations is possible only when the fluid properties reach a statistical equilibrium, for low Reynolds numbers and simple geometries. Unfortunately, the time and space details provided by DNS are not always required for design purposes.

When setting up a problem, near-wall region modeling is important because solid walls are the main source of vorticity and turbulence. Flow separation and reattachment are strongly dependent on a correct prediction of the development of turbulence near walls.

Turbulence modeling starts with following possibilities for definition of the Reynolds stresses in terms on known (averaged) quantities: (1) Boussinesq hypothesis, (2) Reynolds stress transport models, (3) non-linear eddy viscosity models (algebraic Reynolds stress), and (4) model directly the divergence of the Reynolds stresses.

${ }^{*}$ Corresponding author: Karlo T. Raić, karlo@tmf.bg.ac.rs 
The next moment in modeling is the correct determination of the complex velocity profile inside the turbulent boundary layer. Here, a new approach and strategy will be presented.

\section{Structure of the turbulent boundary layer}

For equilibrium turbulent boundary layers, usually, we have the situation presented in Figure 1. [4]. It is well known that at high Reynolds number, the viscous dominated layer is so thin that it is challenging to resolve it.

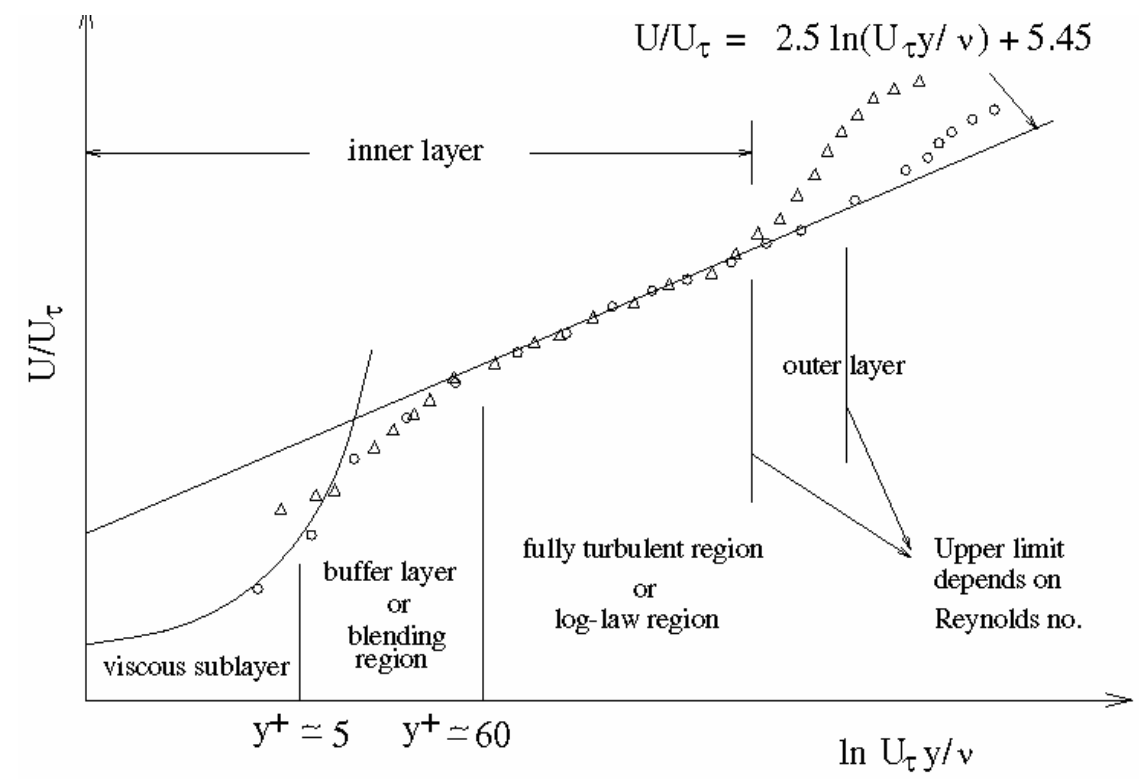

Fig. 1. Dimensionless velocity data from a wide variety of turbulent duct and boundary-layer flows [4].

In Figure 1: $\quad \mathrm{y}^{+}=\frac{\mathrm{y} \mathrm{U}_{\tau}}{v}, \mathrm{u}^{+}=\frac{\mathrm{u}}{\mathrm{U}_{\tau}}, \quad \mathrm{U}_{\tau}=\sqrt{\frac{\tau_{w}}{\rho}}$

where $y$ is the normal distance from the wall, $\tau_{w}$ is wall shear stress, $v$ is kinematic viscosity, $u$ is velocity and $\rho$ is density. 


\section{The mathematical formulation of a complex velocity profile}

Second-order ordinary differential equation from [1], adapted for simulation of complex velocity profile inside the turbulent boundary layer is:

$$
\theta^{\prime \prime}(\xi) \pm c \xi^{n} \pm f(m)=0
$$

where:

$c=f_{1}(N)$ and $n=f_{2}(N)$ : changeable interconnected coefficient and exponent; whole numbers or fractions.

Introducing the relevant notation for turbulent flow, the solution of equation (1) becomes the polynomial:

$$
\theta=N \xi \pm \frac{1}{2} f(m) \xi^{2} \pm(N-1) \xi^{\left[\frac{N}{ \pm(N-1)}\right]^{( \pm 1) *}}
$$

where:

$\theta=\frac{\vartheta-\vartheta_{0}}{\vartheta_{\xi=1}-\vartheta_{0}}$ : normalized dimensionless change of velocity $\vartheta ;$

$\xi=\mathrm{y} / \delta_{\chi}:$ dimensionless distance from surface for position $\chi$;

$\delta_{\chi}$ : boundary layer thickness at position $(\chi)$

$\delta_{\chi}=C_{\chi} \sqrt{\frac{\Pi \chi}{\vartheta_{\xi=1}}}$

$\mathrm{C}_{\chi}$ : coefficient of proportionality;

$\Pi$ : kinematic and/or eddy viscosity $\left[\mathrm{m}^{2} / \mathrm{s}\right]$;

$$
N=\left.\frac{d \theta}{d \xi}\right|_{\xi=0}
$$

Surface Criterion: $\quad d \xi^{\xi=0}$; is whole number or fraction, belongs [0,2];

$\mathrm{N}$ is determined from $\mathrm{m}$ (see ref. [1])

$$
\begin{aligned}
& \qquad f(m)=\left.\frac{d \theta}{d \xi}\right|_{\xi=1} \text {; is whole number or fraction, belongs }[0, \pm \infty] \text {; } \\
& \text { Core Criterion: } \\
& f(m) \text { is determined from } \mathrm{m} \text { (see ref. [1]) }
\end{aligned}
$$

The $\mathrm{m}$ is the whole characteristic number, presents the ratio of formation $(\mathrm{F})$ and decomposition (D) processes inside the boundary layer, $\mathrm{m}=\mathrm{F} / \mathrm{D}= \pm 1,2,3 \ldots$ The quantity $m$ enables the total coupling of the analyzed situation. 
In this approach, three zones exist:

1. Laminar Sublayer (LS)

2. Turbulent Fully developed zone (TF)

3. Turbulent Upper zone (TU)

Every zone has different $\mathrm{m}, \mathrm{N}$, and $f(\mathrm{~m})$ values, with appropriate boundaries $\xi$, Figure 2. and Table 1.

Table 1. An example of the equations set.

\begin{tabular}{llllc}
\hline Zone & $m$ & $N$ & $f(m)$ & Equation \\
\hline $\operatorname{LS}\left[0, \xi_{1}\right]$ & 3 & $1 / 4$ & -1 & $\theta=\frac{3}{4} \xi-\xi^{2}+\frac{1}{4} \xi^{3}$ \\
$\operatorname{TF}\left[\xi_{1}, \xi_{2}\right]$ & 7 & $7 / 8$ & 0 & $\theta=\frac{1}{8} \xi+\frac{7}{8} \xi^{\frac{1}{7}} \approx \xi^{\frac{1}{7}}$ \\
$\mathrm{TU}\left[\xi_{2}, \xi_{3}\right]$ & 5 & $5 / 6$ & $9 / 5$ & $\theta=\frac{1}{6} \xi+\frac{9}{5} \xi^{2}+\frac{5}{6} \xi^{\frac{1}{5}}$ \\
\hline
\end{tabular}

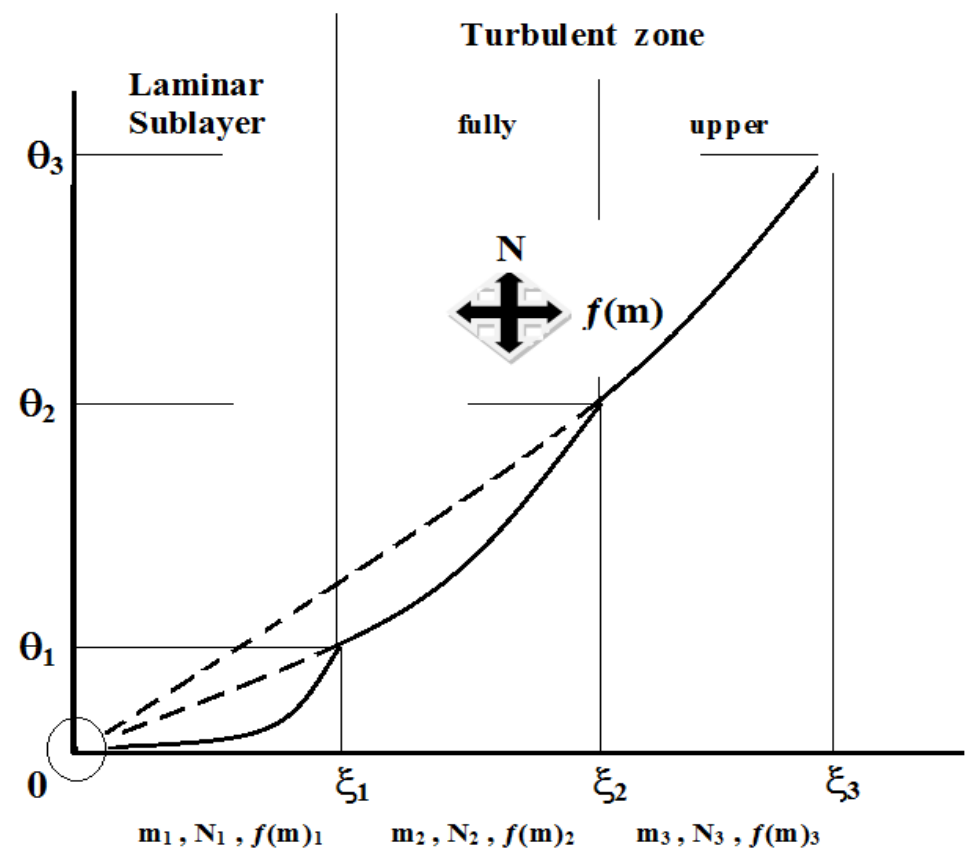

Fig. 2. Scheme of dimensionless complex velocity profile in a turbulent boundary layer.

Directions of actions of $\mathrm{N}$ and $f(\mathrm{~m})$ values on complex velocity profiles in the turbulent boundary layer are indicated in Figure 2. 


\section{Concluding remarks}

Simulation of complex velocity profile in turbulent boundary layer needs a considerable computation ability, usually connected with faulty assumptions. On the other hand, experimental validation is not always a good confirmation for numerical results. Because of that, the new flexible, more simple simulation strategy is suggested.

\section{Acknowledgments}

This work was financially supported by the Ministry of Education, Science and Technological Development of the Republic of Serbia, (Contract No. 451-0368/2020-14/200135).

\section{References}

[1] K.T. Raić: Metall Mater Eng, 24 (2018) 93-102.

[2] H. Schlichting, K. Gersten, Boundary Layer Theory, 8th ed., Springer, 2000, pp. 145-207 and 683-703.

[3] V.N. Saljnikov: Theoretical and Applied Mechanics, 4 (1978) 139-163.

[4] Modeling Turbulent Flows, Ansys Fluent, Introductory FLUENT Notes v6.3, December 2006,

http://www.southampton.ac.uk/ nwb/lectures/GoodPracticeCFD/Articles/Tu rbulence_Notes_Fluent-v6.3.06.pdf

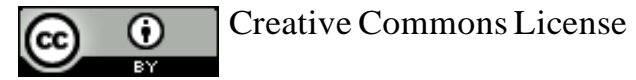

This work is licensed under a Creative Commons Attribution 4.0 International License. 